\title{
O ATENDIMENTO EM LIBRAS COMO GARANTIA DA UNIVERSALIDADE, DA INTEGRALIDADE E DA EQUIDADE NO ACESSO À SAÚDE: UMA REVISÃO NARRATIVA
}

\author{
Bianca Cardoso Lopes'; https://orcid.org/0000-0001-8619-1749; Milene Babeles Viana Silva²; \\ https://orcid.org/0000-0001-6800-5877; Flávia Galvão Hó3; https://orcid.org/0000-0001-9080-5299; Andressa Kênia \\ de Almeida Rodrigues4; https://orcid.org/0000-0002-6932-2177; Kaliani Ângelo Ramos5; https://orcid.org/0000- \\ 0001-7275-9583; Adriana Maria de Figueiredo6; https://orcid.org/0000-0001-9222-6397
}

\section{FILIAÇÃO}

(1) Universidade Federal de Ouro Preto - UFOP, Medical Student

(2) Universidade Federal de Ouro Preto - UFOP, Medical Student

(3) Universidade Federal de Ouro Preto - UFOP, Medical Student

(4) Universidade Federal de Ouro Preto - UFOP, Medical Student

(5) Universidade Federal de Ouro Preto - UFOP, Medical Student

(6) Universidade Federal de Ouro Preto - UFOP, Orientadora

\section{AUTOR CORRESPONDENTE}

Bianca Cardoso Lopes'; https://orcid.org/0000-0001-8619-1749. E-mail da autora principal: biancalopesc50@gmail.com Rua: Santo Antônio №: 447, Bairro: Praia, CEP: 36416-166, Congonhas, Minas Gerais, Brasil.

\section{MENSAGENS-CHAVE}

A LIBRAS é reconhecida na legislação brasileira, mas pouco difundida no meio médico, afetando o acesso à saúde pelos surdos.

A comunicação em LIBRAS assegura a universalidade, ao proporcionar inclusão social dos surdos e respeito aos direitos à saúde.

A LIBRAS atua na garantia da equidade, ao possibilitar um atendimento de qualidade aos surdos, respeitando as singularidades.

O uso da LIBRAS pelos médicos favorece o cuidado integral aos surdos, ao respeitar a individualidade e a autonomia do paciente.

\section{RESUMO}

INTRODUÇÃO: A Língua Brasileira de Sinais (LIBRAS) é a principal comunicação da comunidade surda. Contudo, esta língua ainda é pouco difundida, fomentando o preconceito e a desigualdade sofrida por esta minoria, inclusive na saúde. Neste sentido, o desconhecimento em LIBRAS compromete a assistência aos surdos, pois dificulta a comunicação médico-paciente. Com isso, esta revisão objetiva analisar o uso e o ensino da LIBRAS como garantia de acessibilidade em saúde e cumprimento das diretrizes do Sistema Único de Saúde (SUS). METODOLOGIA: Este estudo trata-se de uma revisão narrativa, elaborada mediante pesquisas científicas, utilizando os descritores: "Acesso aos Serviços de Saúde", "Comunicação", "Surdez", "Educação Médica" e "Sistema Único de Saúde (SUS)". Ademais, foram definidos critérios de inclusão (qualidade metodológica e conteúdo) e exclusão (ausência de palavras-chave relativas ao tema, abordagens exclusivas da surdez e textos duplicados). RESULTADOS: Obteve-se 11 artigos brasileiros dos anos de 2009 a 2020. A partir das pesquisas bibliográficas, as informações foram organizadas em duas tabelas e divididas em: título; autores; ano de publicação; base de dados; nome do periódico; tipo de publicação; palavras-chave, principais resultados e conclusão. DISCUSSÃO: Apesar de a legislação brasileira assegurar atendimento em saúde adequado às pessoas com deficiência auditiva, expressiva parcela dos surdos não recebe os cuidados esperados, devido ao desconhecimento da LIBRAS pelos 
médicos. Destarte, os princípios do SUS são violados e a assistência pensamento crítico, combatendo a desinformação e as "Fake News" relacionadas à vacinação para COVID-19. Então, ficou claro aos surdos é prejudicada. CONCLUSÃO: Diante disso, a LIBRAS mostrou-se fundamental no atendimento humanizado, por respeitar as individualidades dos surdos e assegurar uma boa relação médico-paciente. Sendo assim, evidencia-se a importância do uso e da educação da LIBRAS nos cursos da área da saúde, garantindo, assim, a qualidade da assistência aos surdos e dos princípios do SUS. Por fim, a conscientização cidadã quanto à língua de sinais também é fundamental para a inclusão social efetiva desta minoria.

\title{
PALAVRAS-CHAVE: Acessibilidade aos Serviços de Saúde; Barreiras de Comunicação; Línguas de Sinais; Sistema Único de Saúde (SUS); Surdez.
}

\begin{abstract}
INTRODUCTION: The Brazilian Sign Language (LIBRAS) is the main communication of the deaf community. However, this language is still not widespread, fostering prejudice and inequality suffered by this minority, including in health. In this sense, the lack of knowledge in LIBRAS compromises the assistance to the deaf, because it hinders doctor-patient communication. Thus, this review aims to analyze the use and teaching of LIBRAS as a guarantee of accessibility in health and compliance with the guidelines of the Unified Health System (SUS). METHODOLOGY: This study focused on a narrative review, elaborated through scientific research, using the descriptors: "Access to Health Services", "Communication", "Deafness", "Medical Education" and "Unified Health System (SUS)". Furthermore, inclusion (methodological quality and content) and exclusion (absence of keywords related to the theme, exclusive approaches to deafblindness and duplicate texts) criteria were defined. RESULTS: 11 Brazilian articles from 2009 to 2020. From the bibliographic research, the information were organized in two tables and divided into: title; authors; year of publication; database; journal name; type of publication; keywords, main results and conclusion. DISCUSSION: Although the Brazilian legislation ensures adequate health care to people with hearing loss, a significant portion of the deaf do not receive the expected care, due to the lack of knowledge of LIBRAS by doctors. Therefore, the principles of the Unified Health System are violated, and the assistance to the deaf is impaired. CONCLUSION: In view of this, LIBRAS proved to be fundamental in humanized care, by respecting the individuality of the deaf and ensuring a good doctor-patient relationship. As such, the importance of the use and education of LIBRAS in health courses is evidenced, thus ensuring the quality of care for the deaf and the principles of the SUS. Finally, citizen awareness of sign language is essential for the effective social inclusion of this minority.
\end{abstract}

KEYWORDS: Health Services Accessibility; Communication Barriers; Sign Language; Unified Health System (SUS); Deafness.

\section{INTRODUÇÃO}

No Brasil, a surdez representa uma das deficiências mais prevalentes na população, acometendo milhões de indivíduos em diferentes graus de severidade1. Apesar do número expressivo, o preconceito contra a comunidade de pessoas com deficiência auditiva sempre existiu e as afeta em vários âmbitos, seja academicamente, profissionalmente ou socialmente.

Nesse sentido, é válido destacar que, além de sofrerem com o estigma social, os surdos ainda têm o acesso à saúde prejudicado, haja vista que é raro encontrar um profissional da medicina que saiba se comunicar em Língua Brasileira de Sinais (LIBRAS) - conforme uma pesquisa realizada com profissionais do Sistema Único de Saúde (SUS), cerca de $99 \%$ dos médicos não se comunicam em LIBRAS ${ }^{1}$. Tal paradigma configura uma problemática importante, uma vez que a comunidade surda enfrenta inúmeras barreiras na acessibilidade à saúde - como as falhas de comunicação, que aumentam as chances de diagnósticos equivocados, de erros de prontuário, de constrangimentos, de não adesão ao tratamento, de sofrimento e de insatisfação do usuário ${ }^{2}$. Além disso, a ausência do conhecimento em LIBRAS impacta no atendimento qualificado. Segundo uma pesquisa realizada com pessoas com deficiência auditiva da Associação de Surdos de Maringá (Asumar), a comunicação em LIBRAS com os profissionais da saúde representa a forma mais humanizada do cuidado, sendo considerada relevante para o sentimento de respeito e de conforto durante o atendimento ${ }^{3}$.

Sob tal ótica, percebe-se, no setor de saúde brasileiro, uma dificuldade de alcançar a comunicação efetiva entre os diversos grupos sociais existentes, criando obstáculos culturais e de linguagem ${ }^{3}$. Logo, a falta de adaptação da classe médica para a realidade brasileira dos surdos impede a garantia dos três princípios doutrinários do SUS. Diante desse cenário, a problemática da atenção aos surdos incita a reflexão sobre 0 atendimento em LIBRAS como garantia da universalidade, da integralidade e da equidade no acesso à saúde.

Além disso, esse estudo mostra-se relevante e necessário no contexto atual, no qual existem lacunas no número de evidências, que devem ser preenchidas, visto que há poucas pesquisas e discussões sobre esta temática nos centros acadêmicos. Somado a isso, a abordagem da comunicação em LIBRAS é importante para o planejamento da formação e da preparação dos profissionais de saúde, bem como para o atendimento de qualidade às pessoas com deficiências auditivas.

Por meio de uma revisão narrativa da literatura, este estudo, portanto, objetiva analisar o uso e o ensino da LIBRAS como garantia de acesso à saúde para pessoas com deficiência auditiva. Ademais, este estudo busca discutir as condições vivenciadas pelas pessoas com deficiência auditiva no acesso à saúde e as implicações da falta de comunicação adequada entre médico e paciente. Além disso, esse texto analisa a importância da educação em LIBRAS no contexto da saúde, como forma de assegurar a acessibilidade universal e igualitária para pessoas surdas e não surdas. 


\section{MÉTODOS}

Este estudo trata de uma revisão narrativa realizada nas plataformas MEDLINE/Pubmed e SciELO no período de abril de 2021 a maio de 2021 - período de elaboração da revisão para obter-se a identificação de produções relacionadas ao tema "O atendimento em LIBRAS como garantia da universalidade, da integralidade e da equidade no acesso à saúde". Para o desenvolvimento desta revisão narrativa, foram utilizadas fontes bibliográficas de 2009 a 2020, com predominância de artigos recentes para o reconhecimento do cenário atual.

Durante a elaboração deste artigo, a organização da pesquisa foi sequenciada em: 1) escolha da temática principal e delimitação dos objetivos, com uma pesquisa exploratória para compreensão do assunto; 2) definição das plataformas de busca utilizadas: MEDLINE/Pubmed e SciELO, durante o período de abril de 2021 a maio de 2021; 3) definição dos descritores Decs para as buscas, sendo estes: "Acesso aos Serviços de Saúde", "Comunicação", "Surdez", "Educação Médica" e "Sistema Único de Saúde (SUS)"; 4) uso do operador booleano "OR" para pesquisa utilizando os descritores da seguinte maneira: "Acesso aos Serviços de Saúde" OR "Comunicação", "Surdez" OR "Educação Médica", "Acesso aos Serviços de Saúde" OR "Sistema Único de Saúde (SUS)"; 5) delimitação dos critérios de inclusão: qualidade metodológica e conteúdo do artigo, enquadramento temático (comunicação entre os profissionais da saúde e os surdos por meio do uso de LIBRAS); 6) delimitação dos critérios de exclusão: ausência de palavras-chave relativas a acessibilidade em saúde, pessoas com deficiência auditiva e SUS, artigos que falam exclusivamente da surdez, estudos duplicados; estudos que não foram escritos em português, inglês ou espanhol e estudos datados de anos anteriores a 2009; 7) seleção primária dos artigos nas bases de dados; 8) aplicação dos critérios de inclusão e exclusão; 9) seleção secundária dos artigos após exclusão; 10) análise crítica e seleção final dos artigos.

\section{RESULTADOS}

Após concluir as etapas de pesquisa, foi possível obter 11 artigos, a serem lidos e interpretados criticamente. De acordo com a sequência da metodologia apresentada, os estudos selecionados foram organizados nas Tabelas 1 e 2 . Com relação a esses artigos, é relevante salientar que todos foram publicados no Brasil, uma vez que esta revisão tem como base o estudo dos pilares do SUS e da LIBRAS, os quais têm influência direta para a população brasileira. Dentre os textos escolhidos para análise, uma publicação é de 2020, enquanto uma é de 2019, uma é de 2018, uma de 2017, uma de 2016, uma de 2015, duas de 2013, duas de 2012 e uma de 2009. Da totalidade das publicações, quatro versam sobre a dificuldade do acesso aos serviços de saúde pelos surdos, quatro abordam o uso da linguagem de sinais no atendimento médico, dois analisam a educação médica e a habilidade de comunicação e um discute sobre a humanização da assistência à saúde. Sendo assim, os resultados das buscas por referências permitiram análises socioculturais, informativas e educacionais na área da saúde, vinculadas à percepção e às conexões com o contexto da comunidade surda. As informações contidas na tabela 1 foram categorizadas por: título; autores; ano de publicação; base de dados; nome do periódico; tipo de publicação; palavras-chave, foram extraídas das referências utilizadas. Enquanto na Tabela 2 as informações foram organizadas em título; principais resultados e conclusão.

\section{DISCUSSÃO}

A distinção entre ser surdo e ser uma pessoa com deficiência auditiva está na atuação sociopolítica desempenhada pelo indivíduo. Sendo assim, uma pessoa com deficiência auditiva que luta por seus direitos políticos, linguísticos e culturais faz parte de uma comunidade surda4, a qual se constitui por pessoas com interesses e vivências similares. Neste contexto, a partir das reivindicações políticas desse grupo social, surgiu a Lei $\mathrm{n} \stackrel{0}{ } 10.436$, de 24 de abril de 2002, a qual determina, em seu Art. 3ํㅜ que as instituições de serviços públicos de saúde devem assegurar atendimento e tratamento adequado às pessoas com deficiência auditiva5. Contudo, o cenário dos centros de saúde brasileiros não estão em consonância com a determinação supracitada e com a demanda da população surda, uma vez que significativa parcela dos médicos não possuem conhecimento básico de língua de sinais - de acordo com uma pesquisa realizada em unidades de saúde do SUS, apenas um médico dentre os 101 especialistas entrevistados dominava o uso da LIBRAS para prestar atendimento às pessoas com deficiência auditiva ${ }^{1}$.

Dessa forma, devido à carência de capacitação em língua de sinais para a interação com a comunidade surda, muitos médicos utilizam gestos e mímicas para dar assistência aos surdos6. Tendo como ponto de partida o fato de a surdez não demandar uma acessibilidade facilmente identificável, como é o caso de pessoas com deficiência física, existe uma falsa impressão de que os serviços e profissionais de saúde estão atendendo às necessidades desse grupo de forma satisfatória ${ }^{2}$. No entanto, usar gestuais como forma de comunicação não é eficaz, pois desconsidera os aspectos socioculturais inerentes à primeira língua dos surdos - a LIBRAS $^{5}$. Assim, verifica-se que o vínculo médico-paciente é enfraquecido, prejudicando a qualidade da assistência à saúde ${ }^{7}$. Como consequência, a disfunção no diálogo com a população surda torna inviável a garantia dos três pilares fundamentais do Sistema Único de Saúde - universalidade, equidade e integralidade.

Sob esse viés, é importante analisar como a ausência do uso da LIBRAS pelos médicos interfere especificamente em cada doutrina do Sistema Único de Saúde. Para tanto, faz-se necessário compreender as definições destes princípios e a sua aplicabilidade no contexto da assistência médica aos surdos.

No que concerne à universalidade, salienta-se que este pilar versa sobre o direito de todos à saúde, independentemente de gênero, etnia, idade, classe econômica, profissão, presença ou ausência de deficiência, sem haver qualquer discriminação ou marginalização no atendimento médico ${ }^{8}$. Contudo, na 
Tabela 1. Organização dos artigos, a partir da classificação em: título, autores, ano de publicação, base de dados, nome do periódico, tipo de publicação e palavras-chave

\begin{tabular}{|c|c|c|c|c|c|c|}
\hline Título & Autores & $\begin{array}{l}\text { Ano de } \\
\text { Publicação }\end{array}$ & Base de dados & $\begin{array}{l}\text { Nome do } \\
\text { Periódico }\end{array}$ & $\begin{array}{l}\text { Tipo de } \\
\text { Publicação }\end{array}$ & Palavras-chave \\
\hline $\begin{array}{l}\text { Comunicação } \\
\text { com deficientes } \\
\text { auditivos na ótica } \\
\text { de profissionais } \\
\text { de saúde }\end{array}$ & $\begin{array}{l}\text { Marquete } \\
\text { Verônica } \\
\text { Francisqueti, } \\
\text { Costa Maria } \\
\text { Antônia Ramos, } \\
\text { Teston Elen } \\
\text { Ferraz }\end{array}$ & 2018 & SciELO & $\begin{array}{l}\text { Revista Baiana } \\
\text { de Enfermagem }\end{array}$ & Artigo & $\begin{array}{l}\text { Comunicação; } \\
\text { Perda auditiva; } \\
\text { Pessoal de saúde }\end{array}$ \\
\hline $\begin{array}{l}\text { Conhecimento de } \\
\text { libras pelos } \\
\text { médicos do } \\
\text { Distrito Federal e } \\
\text { atendimento ao } \\
\text { paciente surdo }\end{array}$ & $\begin{array}{l}\text { Gomes L.F, } \\
\text { Machado F.C, } \\
\text { Lopes M.M, } \\
\text { Oliveira R.S, } \\
\text { Medeiros- } \\
\text { Holanda B, Silva } \\
\text { L.B. }\end{array}$ & 2017 & SciELO & $\begin{array}{l}\text { Revista Brasileira } \\
\text { de Educação } \\
\text { Médica }\end{array}$ & Artigo & $\begin{array}{l}\text { Ensino; Pessoas } \\
\text { com Deficiência } \\
\text { Auditiva; } \\
\text { Relações Médico- } \\
\text { Paciente }\end{array}$ \\
\hline $\begin{array}{l}\text { "Meu sonho é ser } \\
\text { compreendido": } \\
\text { Uma análise da } \\
\text { interação médico- } \\
\text { paciente surdo } \\
\text { durante a } \\
\text { assistência à } \\
\text { saúde }\end{array}$ & $\begin{array}{l}\text { Pereira A.A.C, de } \\
\text { Paula Passarin N, } \\
\text { Nishida F.S, } \\
\text { Garcez V.F. }\end{array}$ & 2020 & SciELO & $\begin{array}{l}\text { Revista Brasileira } \\
\text { de Educação } \\
\text { Médica }\end{array}$ & Artigo & $\begin{array}{l}\text { Acesso aos } \\
\text { Serviços de } \\
\text { Saúde; Atenção à } \\
\text { Saúde; } \\
\text { Comunicação em } \\
\text { Saúde; Ensino; } \\
\text { Linguagem de } \\
\text { Sinais; Relações } \\
\text { Médico-Paciente; } \\
\text { Surdez }\end{array}$ \\
\hline $\begin{array}{l}\text { Desafios da } \\
\text { prática do } \\
\text { acolhimento de } \\
\text { surdo na atenção } \\
\text { primária }\end{array}$ & $\begin{array}{l}\text { Tedesco Janaina } \\
\text { dos Reis, Junges } \\
\text { José Roque }\end{array}$ & 2013 & SciELO & $\begin{array}{l}\text { Caderno de } \\
\text { Saúde Pública }\end{array}$ & $\begin{array}{l}\text { Comunicação } \\
\text { Breve }\end{array}$ & $\begin{array}{l}\text { Acesso aos } \\
\text { Serviços de } \\
\text { Saúde; } \\
\text { Acolhimento; } \\
\text { Pessoas com a } \\
\text { Deficiência } \\
\text { Auditiva; Atenção } \\
\text { Primária a Saúde }\end{array}$ \\
\hline $\begin{array}{l}\text { Comunicação } \\
\text { como ferramenta } \\
\text { essencial para } \\
\text { assistência à } \\
\text { saúde dos surdos }\end{array}$ & $\begin{array}{l}\text { Oliveira Yanik, } \\
\text { Carla Araújo de, } \\
\text { Celino Suely } \\
\text { Deysny de Matos, } \\
\text { Costa Gabriela } \\
\text { Maria Cavalcanti }\end{array}$ & 2015 & $\overline{\text { SciELO }}$ & Physis & Artigo & $\begin{array}{l}\text { Comunicação; } \\
\text { Assistência a } \\
\text { saúde; Surdez }\end{array}$ \\
\hline $\begin{array}{l}\text { Dificuldades na } \\
\text { comunicação } \\
\text { entre pessoas } \\
\text { com deficiência } \\
\text { auditiva e } \\
\text { profissionais de } \\
\text { saúde: uma } \\
\text { questão de saúde } \\
\text { pública }\end{array}$ & $\begin{array}{l}\text { Castro Shamyr } \\
\text { Sulyvan de, Paiva } \\
\text { Karina Mary, } \\
\text { César Chester } \\
\text { Luiz Galvão } \\
\end{array}$ & 2012 & SciELO & $\begin{array}{l}\text { Revista da } \\
\text { Sociedade } \\
\text { Brasileira de } \\
\text { Fonoaudiologia }\end{array}$ & Artigo & $\begin{array}{l}\text { Serviços de } \\
\text { Saúde; Perda } \\
\text { auditiva; Pessoas } \\
\text { com deficiência; } \\
\text { Comunicação em } \\
\text { saúde; Equidade } \\
\text { em saúde; } \\
\text { Administração em } \\
\text { saúde }\end{array}$ \\
\hline $\begin{array}{l}\text { Princípios do } \\
\text { sistema único de } \\
\text { saúde (SUS) e a } \\
\text { humanização das } \\
\text { práticas de saúde }\end{array}$ & $\begin{array}{l}\text { Mattos Ruben } \\
\text { Araujo de. }\end{array}$ & 2009 & SciELO & $\begin{array}{l}\text { Interface - } \\
\text { Comunicação, } \\
\text { Saúde, Educação }\end{array}$ & Debate & $\begin{array}{l}\text { Humanização da } \\
\text { assistência; } \\
\text { Integralidade; } \\
\text { Práticas de saúde }\end{array}$ \\
\hline
\end{tabular}




\begin{tabular}{|c|c|c|c|c|c|c|}
\hline Título & Autores & $\begin{array}{l}\text { Ano de } \\
\text { Publicação }\end{array}$ & $\begin{array}{l}\text { Base de } \\
\text { dados }\end{array}$ & $\begin{array}{l}\text { Nome do } \\
\text { Periódico }\end{array}$ & $\begin{array}{l}\text { Tipo de } \\
\text { Publicação }\end{array}$ & Palavras-chave \\
\hline $\begin{array}{l}\text { LIBRAS na } \\
\text { graduação médica: o } \\
\text { despertar para uma } \\
\text { nova língua }\end{array}$ & $\begin{array}{l}\text { Levino, } \\
\text { Danielle de } \\
\text { Azevedo, } \\
\text { Souza, Emyle } \\
\text { Brito de, } \\
\text { Cardoso, } \\
\text { Pedro Capela, } \\
\text { Silva, } \\
\text { Anderson } \\
\text { Carvalho da \& } \\
\text { Carvalho, } \\
\text { Adriana } \\
\text { Edelves } \\
\text { Trindade } \\
\text { Martins }\end{array}$ & 2013 & SciELO & $\begin{array}{l}\text { Revista } \\
\text { Brasileira de } \\
\text { Educação } \\
\text { Médica }\end{array}$ & $\begin{array}{l}\text { Relato de } \\
\text { Experiência }\end{array}$ & $\begin{array}{l}\text { Linguagem de } \\
\text { Sinais; Surdez; } \\
\text { Relações Médico- } \\
\text { Paciente; Acesso } \\
\text { aos Serviços de } \\
\text { Saúde; } \\
\text { Comunicação }\end{array}$ \\
\hline $\begin{array}{l}\text { Desenvolvendo } \\
\text { atitudes, } \\
\text { conhecimentos e } \\
\text { habilidades dos } \\
\text { estudantes de } \\
\text { medicina na atenção } \\
\text { em saúde de pessoas } \\
\text { surdas }\end{array}$ & $\begin{array}{l}\text { Costa Luiza } \\
\text { Santos Moreira } \\
\text { da, Silva } \\
\text { Natália } \\
\text { Chilinque } \\
\text { Zambão da. }\end{array}$ & 2012 & SciELO & $\begin{array}{l}\text { Interface - } \\
\text { Comunicação, } \\
\text { Saúde, } \\
\text { Educação }\end{array}$ & Artigo & $\begin{array}{l}\text { Educação Médica; } \\
\text { Pessoas surdas; } \\
\text { Habilidades de } \\
\text { Comunicação }\end{array}$ \\
\hline $\begin{array}{l}\text { Dificuldades de } \\
\text { profissionais na } \\
\text { atenção à saúde da } \\
\text { pessoa com surdez } \\
\text { severa }\end{array}$ & $\begin{array}{l}\text { Gil de França } \\
\text { Euripedes, } \\
\text { Andrade } \\
\text { Pontes Maiary, } \\
\text { Cavalcanti } \\
\text { Costa Gabriela } \\
\text { Maria, Sátiro } \\
\text { Xavier de } \\
\text { França Inácia }\end{array}$ & 2016 & SciELO & $\begin{array}{l}\text { Ciencia y } \\
\text { enfermeria }\end{array}$ & Investigação & $\begin{array}{l}\text { Surdez; pessoal de } \\
\text { saúde; Atenção à } \\
\text { saúde; Barreiras de } \\
\text { Comunicação; } \\
\text { Enfermagem }\end{array}$ \\
\hline $\begin{array}{l}\text { Perceptions of deaf } \\
\text { subjects about } \\
\text { communication in } \\
\text { Primary Health Care }\end{array}$ & $\begin{array}{l}\text { Santos A.S, } \\
\text { Portes, A.J.F }\end{array}$ & 2019 & PubMed & $\begin{array}{l}\text { Revista Latino- } \\
\text { Americana de } \\
\text { Enfermagem }\end{array}$ & Artigo & $\begin{array}{l}\text { Acessibilidade; } \\
\text { Atenção Primária à } \\
\text { Saúde; Barreiras de } \\
\text { Comunicação; } \\
\text { Comunicação; Perda } \\
\text { de audição; Surdez }\end{array}$ \\
\hline
\end{tabular}

Fonte: Própria 
Tabela 2. Organização dos artigos, a partir da classificação em: título, principais resultados e conclusões

\section{Título}

ótica de profissionais de saúde

\section{Principais Resultados}

Verificou-se que $92,4 \%$ dos profissionais acreditavam estar despreparados para atender o deficiente auditivo, 83,8\% não sabiam comunicar-se com esses e $96,5 \%$ não sabiam se comunicar em Libras. Muitos utilizam estratégias visando quebrar a barreira de comunicação, tais como: gestos e escritas (18,7\%), fala e gestos $(11,6 \%)$, entre outros.

Conhecimento de libras pelos médicos do Foram entrevistados médicos de 24 Distrito Federal e atendimento ao paciente especialidades, com idade média de 41 surdo anos. Deles, $92,1 \%$ já atenderam um paciente surdo e $76.2 \%$ consideraram o conhecimento de Libras importante para sua prática médica, mas apenas um relatou conhecimento básico na língua. Quanto ao sentimento do médico no atendimento, houve predomínio de incerteza e desconforto. Um número significativo de médicos já realizou atendimento de pacientes surdos em sua prática profissional no SUS, e a maioria considerou o conhecimento de Libras relevante, especialmente os médicos com menos de 55 anos de idade.

\section{Conclusões}

Na percepção dos profissionais de saúde, a barreira de comunicação com os deficientes auditivos ocorria por não saberem comunicar-se em Libras; por isso, utilizavam mecanismos como gestos e mímicas para tentar a comunicação

Possivelmente, o sentimento de desconforto no atendimento decorre do predominante desconhecimento da língua pelos médicos e da conseguinte dificuldade durante $o$ atendimento. Destaca-se a importância da implantação ou ampliação do estudo de Libras antes ou durante a formação médica e dos demais cursos da área da saúde. $\mathrm{A}$ conscientização dos profissionais de saúde perante $o$ atendimento integral do paciente surdo é um passo fundamental na implementação efetiva do ensino de Libras de forma especializada no ensino superior, resultando em maior confiança e qualidade na relação médico-paciente

\begin{abstract}
"Meu sonho é ser compreendido": Uma análise da interação médico-paciente surdo durante a assistência à saúde
\end{abstract}

Dentre os médicos e acadêmicos, 76\% afirmaram que já atenderam um paciente com surdez grave parcial ou severa. Embora 49\% dos surdos tenham afirmado que já sentiram algum desconforto e também alguma segurança no atendimento, 55,5\% mencionaram que já deixaram de ir ao médico por medo de não serem compreendidos ou relataram algum problema, como dor, desconforto ou angústia
Desafios da prática do acolhimento de surdo na atenção primária

Os resultados evidenciaram que os profissionais buscam diferentes ferramentas para obviar a dificuldade da comunicação com os surdos, e que a postura dos profissionais em geral manifesta desconforto e despreparo para atender às necessidades dos portadores de surdez

Comunicação como ferramenta essencial para assistência à saúde dos surdos

\section{Não obtiveram sucesso no alcance do} entendimento pleno da comunicação e, com o fracasso do método oralista, aceitaram aprender a Libras e hoje é a forma predominante e mais eficiente de estabelecer sua comunicação, exaltando necessidade dos surdos se comunicarem em língua de sinais e participarem ativamente das decisões sobre sua vida
As percepções dos diferentes atores da interação médico-paciente analisados mostraram diferença de satisfação com o serviço e riscos à saúde dos surdos, o que significa que falta planejamento multimodal com estratégias de comunicação efetivas

Se o acolhimento compreende ao mesmo tempo ferramentas adequadas de comunicação e postura ética de escuta qualificada, os resultados da pesquisa demonstram que existem deficiências no acolhimento

As estratégias de comunicação com os usuários surdos adotados pelos profissionais de saúde se mostraram ineficientes e mesmo a presença do acompanhante como interlocutor não foi suficiente para garantir uma assistência de qualidade, visto que os surdos se mostraram sujeitos passivos no seu próprio processo saúde-doença 


\begin{tabular}{ll}
\hline Título & Principais Resultados \\
\hline Comunicação como ferramenta essencial & Não obtiveram sucesso no alcance do \\
para assistência à saúde dos surdos & $\begin{array}{l}\text { entendimento pleno da comunicação e, } \\
\text { com o fracasso do método oralista, }\end{array}$
\end{tabular}
aceitaram aprender a Libras e hoje é a Conclusões

As estratégias de comunicação com os usuários surdos adotados pelos profissionais de saúde se mostraram ineficientes e mesmo a presença do forma predominante e mais eficiente de acompanhante como interlocutor não foi estabelecer sua comunicação, exaltando a suficiente para garantir uma assistência necessidade dos surdos se comunicarem de qualidade, visto que os surdos se em língua de sinais e participarem mostraram sujeitos passivos no seu ativamente das decisões sobre sua vida

Dificuldades na comunicação entre pessoas com deficiência auditiva e profissionais de saúde: uma questão de saúde pública
Dos entrevistados, 35\%\% relataram problemas para ouvir e entender os profissionais de saúde no último serviço visitado; 30,6\% (IC 95\%: 23,4-37,8) para entender os médicos; $18,1 \%$ (IC 95\%: 12,0-24,1) para entender as enfermeiras; e $21,2 \%$ (IC95\%: 14,8-27,6) para entender os outros funcionários. Não houve diferenças quando se considerou as variáveis demográficas, a necessidade de auxílio para tomar banho e se vestir, comer, levantar-se e/ou andar, possuir ou não plano privado de saúde e tipo de serviço de saúde visitado.

Princípios do sistema único de saúde (SUS) e a humanização das práticas de saúde
Apresenta as relações entre os princípios do SUS e a perspectiva de transformações das práticas em saúde, abordando os conceitos das diretrizes do sistema de saúde e sua aplicação próprio processo saúde-doença

Dos entrevistados, 35\%\% relataram problemas para ouvir e entender os profissionais de saúde no último serviço visitado; 30,6\% (IC 95\%: 23,4-37,8) para entender os médicos; $18,1 \%$ (IC 95\%:

12,0-24,1) para entender as enfermeiras; e $21,2 \%$ (IC95\%: 14,8-27,6) para entender os outros funcionários. Não houve diferenças quando se considerou as variáveis demográficas, a necessidade de auxílio para tomar banho e se vestir, comer, levantar-se e/ou andar, possuir ou não plano privado de saúde

O texto reconhece que, apesar da heterogeneidade do movimento sanitário, a questão da transformação das práticas de saúde tem se tornado mais importante para a sustentabilidade e legitimidade do SUS. Partindo do exame dos princípios da universalidade e equidade examinados ao lado do princípio da integralidade, o autor defende que a categoria do sofrimento, manifesto ou não, deve ser incluída nas políticas que se voltem para a construção de práticas mais humanizadas no SUS O minicurso ministrado transpôs barreiras no ensino curricular dos alunos participantes e proporcionou ampliação do conhecimento sobre a temática. A relevância social do projeto deu-se em âmbito acadêmico e social por contribuir para a formação dos alunos; facultar a oportunidade de obtenção de novos conhecimentos; suscitar reflexões por parte dos discentes sobre a cultura surda, favorecendo a inclusão destes; e destruir estigmas e preconceitos sobre a comunidade surda 


\begin{tabular}{ll}
\hline Título & Principais Resultados \\
\hline Desenvolvendo atitudes, conhecimentos & Foram utilizadas categorias e \\
e habilidades dos estudantes de & subcategorias que foram apresentadas \\
medicina na atenção em saúde de & utilizando-se trechos dos relatos dos \\
pessoas surdas & estudantes para exemplificá-las
\end{tabular}

Conclusões

As escolas médicas não deveriam mais formam profissionais que desconhecessem as necessidades de saúde de pessoas com deficiência, as formas alternativas de comunicação com pessoas não verbais, e os riscos que essa lacuna pode gerar.

Dificuldades de profissionais na atenção à saúde da pessoa com surdez severa
Dentre as dificuldades, destacaram-se: comunicação prejudicada, déficit na formação de recursos humanos para a consulta e reconhecimento das necessidades de saúde, infraestrutura inadequada para acolhimento e atendimento ao surdo, incerteza com relação aos cuidados em saúde prescritos na consulta e prejuízo da autonomia do paciente

Perceptions of deaf subjects
communication in Primary Health Care

\begin{abstract}
A falta de intérpretes e a falta de uso da Língua Brasileira de Sinais pelos profissionais foram percebidos como as principais barreiras de comunicação. Por sua vez, a presença de acompanhantes ouvintes (73\%) e o uso de mímica/gestos $(68 \% \%)$ estiveram entre as estratégias mais utilizadas pelos surdos. A maioria dos surdos relatou insegurança nas consultas, e os que melhor compreenderam seu diagnóstico e tratamento foram os surdos bilíngues ( $p=0,0347)$ e os surdos que utilizavam comunicação oral $(p=0,0056)$
\end{abstract}

\section{Percebeu-se que a comunicação} prejudicada constitui uma barreira para a promoção de saúde e que profissionais e unidades de saúde não estão capacitados para acolher e atender às necessidades de saúde da pessoa com surdez severa

\begin{abstract}
A comunicação com os profissionais era facilitada quando os surdos tinham acompanhante ou quando faziam uso de mímica e gestos. A língua de sinais foi negligenciada, apesar de o atendimento ao surdo por profissionais capacitados para o uso dessa língua ser garantido pela legislação
\end{abstract}

\section{Fonte: Própria}

realidade de significativa parcela das pessoas com deficiência auditiva, este princípio é violado, à medida que a falta de comunicação em LIBRAS prejudica a assistência às necessidades dos pacientes.

Perante o desconhecimento da Língua Brasileira de Sinais, muitos médicos utilizam-se de gestos e mímicas para o atendimento à população surda. Entretanto, diferentemente da LIBRAS, que é considerada uma língua espaço-visual natural, com critérios linguísticos e com expressões faciais e corporais bem estabelecidas ${ }^{4}$, os gestos manuais usados socialmente não seguem um padrão específico, bem como não são suficientes para manifestar a intensidade e a entonação das frases. Dessa forma, a relação médicopaciente é afetada, pois a mímica não abrange a totalidade do que os profissionais da saúde desejam informar aos surdos e não expressa todas as dores e sentimentos dos pacientes, interferindo na qualidade da assistência médica, no reconhecimento da subjetividade dos indivíduos, nos diagnósticos e na resistência aos tratamentos pelas pessoas com deficiência auditiva ${ }^{5}$. Assim, nota-se que o desconhecimento da LIBRAS viola o pilar da universalidade, uma vez que atua como ferramenta de exclusão social, ao impossibilitar que o paciente surdo receba o cuidado em saúde esperado e tenha todas as suas necessidades de assistência asseguradas. Além disso, a dificuldade do contato direto entre médico e paciente, muitas vezes, inibe a procura dos indivíduos surdos pelos centros de saúde9, devido aos constrangimentos durante a consulta, ao medo, à frustração, às falhas na interpretação e aos problemas na expressão das dores e dos sintomas ${ }^{7}$.

Já no que tange à equidade, vale ressaltar que este princípio refere-se ao tratamento dos pacientes, de modo que os indivíduos em condições diferentes devem ser atendidos de modo distinto e as pessoas em circunstâncias iguais devem ser tratadas de forma igual, compreendendo as particularidades no cuidado aos pacientes a partir das necessidades de saúde individuais8. Nesse sentido, cabe dizer que o paciente surdo e 0 não surdo se encontram em situações diferentes, visto que o primeiro necessita de um médico capaz de reconhecer suas enfermidades e demandas utilizando a LIBRAS, enquanto o segundo não utiliza esta linguagem. Frente a essa diferença, a assistência à saúde deveria abarcar os dois grupos de pacientes, usando comunicações distintas para reconhecer as particularidades e para respeitar a diversidade sociocultural que envolve 0 processo linguístico.

Ademais, verifica-se que a ausência de capacitação básica em LIBRAS impede o cumprimento da equidade, tendo em vista que as diferenças entre os surdos e os não surdos não é 
considerada, o que torna o cuidado às pessoas com deficiência auditiva ineficiente. Sendo assim, é válido destacar que a própria graduação médica é falha na preparação de um profissional capaz de assegurar a equidade, pois, diferentemente dos cursos de licenciatura e de fonoaudiologia, nos quais o aprendizado em LIBRAS é obrigatório, para a medicina, a língua de sinais permanece como um componente curricular facultativo ${ }^{5}$. Com isso, diversos médicos completam a faculdade sem se capacitar em LIBRAS, encontrando barreiras de comunicação com os surdos no sistema de saúde brasileiro. Conclui-se que, sem informações de saúde acessíveis e sem atendimento médico eficaz, a vulnerabilidade das pessoas com deficiência auditiva às doenças e ao autocuidado aumentam, uma vez que a inexistência de preparação para a assistência singular às pluralidades socioculturais e aos grupos minoritários inibem a plena inserção dos surdos nos programas de saúde ${ }^{10}$.

Por fim, no tocante à integralidade, faz-se necessário compreender que, segundo este princípio doutrinário do SUS, para uma assistência integral, o médico deve reconhecer seu paciente a partir de uma visão holística ${ }^{8}$, que considera o bem-estar físico e mental da pessoa, assim como o contexto sociocultural no qual se insere. Destarte, reconhecendo os fatores que interferem na qualidade de vida e no bem-estar completo dos indivíduos, o sistema de saúde deve garantir todas as formas de assistência, as quais englobam a prevenção, a promoção e a reabilitação da saúde. No entanto, a ausência do uso de LIBRAS pelos médicos prejudicam o cumprimento da integralidade no atendimento da comunidade surda, pois sem 0 reconhecimento da língua do paciente - a qual carrega sua cultura, suas vivências, sua visão de mundo e sua particularidade4 - não é possível oferecer da melhor maneira todos os níveis de cuidado ao paciente.

Assim, para garantir uma assistência integral, é fundamental fundamental que o médico compreenda a cultura, o contexto e a individualidade da pessoa surda. De acordo com o Censo brasileiro de 2000, a população com algum tipo de deficiência apresenta taxas de escolarização, de ocupação e de renda menores que os índices das pessoas sem nenhuma deficiência ${ }^{4}$. Nesse sentido, verificase que, além da língua diferente, o estilo de vida e as condições socioeconômicas da comunidade surda são muito particulares para este grupo social, devendo ser consideradas durante um atendimento médico. Entretanto, sem o reconhecimento da LIBRAS e das questões sociais e culturais inerentes à língua, a assistência à saúde dos surdos torna-se falha e insuficiente para compreender todas as demandas dos pacientes e modificar o cenário de desigualdade presente na vivência da comunidade surda.

Somado a isso, ressalta-se que a problemática na comunicação e na relação entre médico e paciente surdo interfere nos processos de cuidado - tanto de prevenção quanto de tratamento. Sendo assim, usar a escrita como meio de comunicação, falar alto ou gesticulado para dialogar com o paciente surdo, mostrar-se impaciente no processo comunicativo e desconhecer a LIBRAS demonstram despreparo profissional dos médicos na assistência e incompreensão da identidade e da cultura da comunidade surda. Nesse contexto, verifica-se a dificuldade no cumprimento da integralidade ${ }^{3}$, a qual deve considerar a independência, a individualidade, o contexto sociocultural e a privacidade do paciente durante a assistência à saúde. Diante disso, cabe dizer que, considerando a expressiva parcela dos profissionais da saúde que não usam a LIBRAS, frequentemente muitos pacientes recorrem a amigos $\mathrm{e}$ familiares que dominam a língua de sinais como acompanhantes para ir às unidades de saúde e para garantir um entendimento entre médico e usuário. Apesar da relevância do acompanhante na mediação da comunicação, a presença de pessoas conhecidas durante a consulta médica viola a privacidade do paciente surdo6, que precisa relatar sua experiência individual, suas dores e suas dúvidas a um parente ou a um amigo, expondo alguns aspectos íntimos. Como consequência, a presença de um acompanhante pode inibir a manifestação das queixas pelos usuários surdos, devido ao constrangimento em abordar alguma questão de saúde, impactando o modo como o médico irá tratar o paciente ou fornecer orientações para a promoção de uma qualidade de vida.

Além disso, a necessidade recorrente de familiares e de amigos para interpretar e mediar a relação médico-paciente impede a autonomia dos surdos durante a assistência médica e o autocuidado ${ }^{11}$, desrespeitando a individualidade de cada paciente. Somado a isso, a mediação da comunicação pode interferir na interpretação do acompanhante e do médico a respeito dos sintomas sentidos pelo paciente surdo ${ }^{6}$,os quais são singulares e compreendidos da melhor maneira quando informado de forma direta, sem qualquer interferência no diálogo. Dessa forma, a pessoa com deficiência auditiva se sente passiva no cuidado à saúde, comprometendo a relação de confiança entre médico e paciente ${ }^{7}$, assim como prejudicando os processos de prevenção, reabilitação e promoção à saúde por limitar o diálogo e o questionamento direto na assistência.

Por fim, considerando o baixo número de bibliografias encontradas relativas ao uso de LIBRAS no atendimento em saúde, nota-se que esta revisão possui limitações quanto à sistematização e à impossibilidade de replicação metodológica. Com isso, verifica-se a crescente necessidade de abordar este assunto nos centros de saúde e no meio universitário, a fim de ampliar os debates e as intervenções sobre essa problemática, que impacta o cumprimento das diretrizes do SUS. Assim, a partir das referências escolhidas e da análise crítica, foi possível compreender a perspectiva das equipes de saúde com relação ao atendimento em LIBRAS e às experiências individuais de pacientes surdos, reforçando a importância da comunicação eficaz na assistência integral e universal, respeitando a equidade.

\section{CONCLUSÃO}

Conclui-se, com esse estudo, que o atendimento em LIBRAS é fundamental para a garantia dos três pilares do SUS universalidade, equidade e integralidade - e do acesso à saúde. Desse modo, sem o devido conhecimento linguístico, a 
prestação da assistência às pessoas surdas se torna extremamente comprometida, por não atender às demandas singulares deste grupo social, como a comunicação e às especificidades da identidade sociocultural dessa comunidade.

Além disso, foi possível discutir as condições vivenciadas pelas pessoas com deficiência auditiva no acesso à saúde, constatando-se a fragilidade do SUS no que tange ao atendimento eficaz, que respeite a autonomia $e$ a individualidade dos pacientes, sendo necessária a adoção de medidas que possam reverter essa realidade. Portanto, para que se compreenda a relevância da LIBRAS na educação em saúde, sugere-se que o aprendizado da Língua Brasileira de Sinais seja configurado como disciplina de caráter obrigatório em todos os cursos da área da saúde, assegurando a universalidade e a equidade no acesso aos serviços médicos e aos atendimentos nos ambulatórios, nas clínicas e nos hospitais.

Ademais, deve-se estimular a criação de projetos de extensão nas universidades que pesquisem e analisem o atendimento às pessoas com deficiência auditiva e, ainda, que possam atuar revertendo essa realidade do descaso quanto a estes pacientes na atenção à saúde.

Por fim, é importante a atuação governamental no fornecimento de cursos gratuitos de LIBRAS, os quais possam abranger toda a população, a fim de que a língua de sinais se torne mais conhecida pelos brasileiros, possibilitando que essas pessoas sejam compreendidas e inseridas em todos os âmbitos sociais e não somente no cenário médico.

\section{CONFLITO DE INTERESSE}

As autoras declaram que não há nenhum conflito de interesse presente no estudo.

\section{FINANCIAMENTO}

As autoras declaram que não houveram fontes de financiamento.

\section{REFERÊNCIAS}

1. Gomes LF, Machado FC, Lopes MM, Oliveira RS, Medeiros-Holanda B, Silva LB, et al. Conhecimento de Libras pelos Médicos do Distrito Federal e Atendimento ao Paciente Surdo. Revista Brasileira de Educação Médica [Internet]. 2017 Dez; 41(4):551-6. Disponível em: http://www.scielo.br/j/rbem/a/xWSdRHms6gthB4YzWgpZf $\underline{\mathrm{wt} / \text { ?lang=pt }}$

2. Costa LSM, Silva NCZ. Desenvolvendo atitudes, conhecimentos e habilidades dos estudantes de medicina na atenção em saúde de pessoas surdas. Interface Comunicação, Saúde, Educação [Internet]. 2012 Out; 16(43):1107-17. Disponível em: http://www.scielo.br/j/icse/a/DPSPwqgsb3jYXTBQ7xqykqL/ abstract/?lang=pt
3. Pereira AAC, Passarin NP, Nishida FS, Garcez VF. "Meu Sonho É Ser Compreendido": Uma Análise da Interação Médico-Paciente Surdo durante Assistência à Saúde. Revista Brasileira de Educação Médica [Internet]. 2020 Ago; 00;44(4). Disponível em: http://www.scielo.br/j/rbem/a/jWsw9bn6YC8Lj3C6Wxp4 8LB/abstract/?lang=pt

4. Levino DA, Souza EB, Cardoso PC, Silva AC, Carvalho AETM. Libras na graduação médica: o despertar para uma nova língua Libras na graduação médica: o despertar para uma nova língua [Internet]. 2013 Ago; 37(2), 291-297. Disponível em: https://www.scielo.br/j/rbem/a/gYMdRfpj44CZ9WfWS5 MKyXk/?lang=pt

5. Marquete VF, Costa MAR, Teston EF. Comunicação com deficientes auditivos na ótica de profissionais de saúde. Revista Baiana de Enfermagem [Internet]. 2018 Mar 20; 32(0). Disponível em: https://periodicos.ufba.br/index.php/enfermagem/article/ view/24055

6. Oliveira YCA, Celino SDM, Costa GMC. Comunicação como ferramenta essencial para assistência à saúde dos surdos. Physis: Revista de Saúde Coletiva [Internet]. 2015; 25(1):307-20. Disponível em: http://www.scielo.br/j/physis/a/XnMSZYLXkdcx8z7kFBX 3Bpz/abstract/?lang=pt

7. Castro SS, Paiva KM, César CLG. Dificuldades na comunicação entre pessoas com deficiência auditiva e profissionais de saúde: uma questão de saúde pública. Revista da Sociedade Brasileira de Fonoaudiologia [Internet]. 2012 Jun; 17(2):128-34. Disponível em: http://www.scielo.br/j/rsbf/a/ML37tNjJFtXCZjRq4Dy7rqR /abstract/?lang=pt

8. Mattos RA. Princípios do Sistema Único de Saúde (SUS) e a humanização das práticas de saúde. Interface - Comunicação, Saúde, Educação [Internet]. 2009; 13(Suppl.1):771-80. Disponível em: http://www.scielo.br/j/icse/a/GCvzrJvLB3rq8cnvRQpPC $\underline{9 H / \text { ?lang }=p t . ~}$

9. Tedesco JR, Junges JR. Desafios da prática do acolhimento de surdos na atenção primária. Cadernos de Saúde Pública [Internet]. 2013 Ago; 29(8):1685-9. Disponível

em: http://www.scielo.br/j/csp/a/5Y3x8GJpdRBzmfFwdMWT Ddc/abstract/?lang $=\mathrm{pt}$

10. Santos AS, Portes AJF. Perceptions of deaf subjects about communication in Primary Health Care. Revista latino-americana de enfermagem [Internet]. 2019 Mar; $27 . \quad$ Disponível em: https://pubmed.ncbi.nlm.nih.gov/30916228/ 
11. França EG, Pontes MA, et al. Dificuldades de profissionais da atenção à saúde da pessoa com surdez severa. Ciencia y enfermería [Internet]. 2016 Sep; 22(3):107-16. Disponível em: http://www.scielo.cl/scielo.php?script=sci arttext\&pid=S 0717-95532016000300107\&lng=es\&nrm=iso\&tlng=pt. 